CARTA AL EDITOR

Rev Chil Salud Pública 2020,

Vol 24(2) 167-168

Claudia Troncoso-Pantoja

Centro de Investigación en Educación y Desarrollo, Universidad Católica de la Santísima Concepción

(CIEDE-UCSC), Departamento de Salud Pública, Facultad de Medicina, Universidad Católica de la Santísima

Concepción, Concepción, Chile.

Núcleo Científico Tecnológico

para el Borde Costero Sustentable, Universidad Católica de la Santísima

Concepción, Concepción, Chile. ctroncosop@ucsc.cl

Ana María Leiva-Ordoñez Instituto de Anatomía, Histología y Patología, Facultad de Medicina,

Universidad Austral de Chile, Valdivia, Chile.

María Adela Martínez-Sanguinetti Instituto de Farmacia, Facultad de Ciencias,

Universidad Austral de Chile, Valdivia, Chile.

Fanny Petermann-Rocha Institute of Health and Wellbeing, University of Glasgow, Glasgow, United Kingdom.

BHF Glasgow Cardiovascular Research Centre, Institute of

Cardiovascular and Medical Sciences, University of Glasgow, Glasgow, United Kingdom.

Eduard Maury- Sintjago Departamento de

Nutrición y Salud Pública,

Facultad Ciencias de la Salud y de los Alimentos,

Universidad del Bío-Bío, Chillán, Chile.

\section{UN APORTE A LA COMPRENSIÓN DE LOS AMBIENTES ALIMENTARIOS DOMÉSTICOS EN PERSONAS MAYORES}

\author{
A CONTRIBUTION TO UNDERSTANDING DOMESTIC FOOD \\ ENVIRONMENTS IN OLDER PEOPLE
}

\section{Sr. Editor:}

En la edición $24^{1}$ del año 2020 de esta revista, Araneda y cols. presentaron una importante actualización a la temática de los entornos o ambientes alimentarios (AA), aportando definiciones desde diversas fuentes de información de los AA, su aplicación en el campo del conocimiento y una reflexión sobre su utilización para el logro de una alimentación saludable. ${ }^{1}$

Como aporte a la comprensión de este tema, el AA doméstico o domiciliario es uno de los entornos alimentarios que presenta una importancia fundamental en el estudio de la alimentación, puesto que el hogar es el lugar donde se consumen la mayor parte de los alimentos durante el día. ${ }^{2}$ El comer en casa es una de las acciones más comunes de las actividades que realiza una persona, ${ }^{3}$ entregando un simbolismo con formas y significados que incluyen la valoración de un plato desde la persona que lo elaboró, con los afectos y significados de quien lo prepara y para quien va dirigido. Las preparaciones culinarias "hechas con sus propias manos", entregan afectos y tradiciones socioculturales, que le dan una trascendencia a la persona que la consume. ${ }^{4}$ La tradición pasa a ser determinante en la comida diaria, convergiendo las preferencias o aversiones alimentarias en la forma en que se preparan los platos culinarios, creando esta última acción, una oportunidad para una alimentación más saludable ${ }^{5}$. Sin embargo, la globalización y la economía de libre mercado han modificado los patrones alimentarios, aportando desde las sociedades de consumo a la alimentación, estereotipos para cumplir con el yo ideal. ${ }^{6}$

A lo anterior y para comprender de mejor manera los AA domésticos, se debe profundizar en el por qué las personas comen lo que comen, los ritos que mantienen y el significado que se le atribuye al comer. ${ }^{7}$ El sentido de la comida y del comer en AA domésticos convergen en dimensiones que permite explicar que se cocina para la familia, con rituales que se mantienen en el tiempo y que permiten entregar afectos, cuidados, mantener tradiciones, resaltar a los ancestros, dar identidad de género; en resumen, celebrar desde el significado y valoración que se le entrega a un plato preparado. ${ }^{4}$ La comensabilidad favorece la cohesión, los vínculos y las alianzas emocionales, además de las relaciones parentales y la identidad colectiva. ${ }^{8}$

En el AA doméstico y en especial para las personas mayores, la alimentación construye lazos emocionales y sociales, como también de cuidado de la salud, originado desde la preparación de la comida casera, a partir de momentos de entrega de afectos y emociones. ${ }^{9}$ De esta forma, el plato que llega a la mesa, es concientizado por quien lo prepara en otra persona o un grupo familiar, considerando preferencias o gustos personales al momento de adquirir ingredientes, elaborar, servir y consumir un plato culinario. ${ }^{5}$ Consecuentemente, las comidas caseras preparadas por personas mayores, permiten mantener tradiciones familiares y sociales en torno a la alimentación. La elaboración de un plato culinario con significado emocional y afectivo para la persona que la prepara evoca recuerdos, que permiten un traslado 
en el tiempo respecto de situaciones y personas significativas en la vida, además de activar la memoria gustativa a partir del recuerdo del sabor y aroma de los platos y los momentos que son generalmente positivos para la persona mayor. ${ }^{10}$

La compleja visión que nos deja la actual crisis sanitaria y su repercusión socioeconómica, puede ser una oportunidad de revalorar los AA domésticos, como un espacio para entrelazar la cultura alimentaria, las comidas tradicionales y los alimentos naturales con el saber culinario de las personas mayores.

\section{REFERENCIAS BIBLIOGRÁFICAS}

1. Araneda J, Pinheiro AC, Rodríguez L. Una mirada actualizada sobre los ambientes alimentarios y obesidad. Rev Chil Salud Pública. 2020; 24(1): 67-71.

2. Cerda R, Egaña D, Gálvez P, Masferrer D. Marco conceptual sobre factores condicionantes de los ambientes alimentarios en Chile [en línea]; 2016 [consultado en Agosto de 2020]. Disponible en: http://www.bibliotecaminsal.cl/marco-conceptual-sobre-los-factores-condicionantes-de-los-ambientes-alimentarios-en-chile/

3. Barrial AM, Barrial AM. La Educación alimentaria y nutricional desde una dimensión sociocultural como contribución a la seguridad alimentaria y nutricional [en línea]; 2011 [consultado en Agosto de 2020]. Disponible en: https://www.eumed.net/rev/cccss/16/ bmbm.html
4. Hernández M. A la altura de lo cotidiano: algunos sentidos del cocinar y comer en casa. Fundam Humanid. 2008; 9(2): 71-90.

5. Troncoso-Pantoja C, Alarcón-Riveros M, Amaya-Placencia A. Significado emocional de la alimentación en personas mayores. Rev Fac Med. 2019; 67(1): 51-55.

6. Denegri M, Knabe Y, Sepúlveda J, Orellana L, Ruiz O, Vivallo $\mathrm{O}$, et al. Socialización y transmisión intergeneracional en pautas de consumo de alimentos: su impacto en la identidad cultural alimentaria en mujeres mapuches urbanas de Chile. RA XIMHAI. 2016; 12(4): 57-70.

7. Jäger M, Feito M, Le Gall J, Vera N, Martínez R, Castellanos V, et al. Estudio alimentario sobre la comensalidad en la ciudad autónoma de Buenos Aires y su periurbano. Nadir: Rev Elect Georg Austral. 2018; 10(2): 1-27.

8. Maury E. Ritos de comensalidad y espacialidad. Un análisis antropo-semiótico de la alimentación. Gaz Antropol. 2010; 26(2): 45.

9. Troncoso C. Patrimonio gastronómico en personas mayores. Rev Hum Med. 2018; 18(2): 171-75.

10. Troncoso C, Burdiles G, Petermann F. Significado de las comidas tradicionales para mujeres adultas mayores de la provincia de Concepción, Chile. Rev Esp Nutr Comunitaria. 2020; 26(1): e19776. 